SELF-NEGLECT: A FINE BALANCE FOR PROFESSIONALS

P. Leahy-Warren, H. Mulcahy, G. McCarthy Haslam, M. Day, School of Nursing \& Midwifery, University College Cork, Cork, Cork, Ireland

Self-neglect $(\mathrm{SN})$ is a serious public health issue that is largely hidden. Ageing demography will

increases the significance of self-neglect for community professionals. The aim of this qualitative study was to explore perceptions of self-neglect among Community Nurses (CNs), Public Health Nurses (PHNs), and Social Workers (SWs). Open-ended questions, reported here were part of a large quantitative cross -sectional descriptive study. From overall sample of 566, recruited from community health organisation in Ireland, $25.6 \%(n=87)$ of participants responded. The framework method was used for analysing the cross-sectional descriptive data. Findings revealed one overarching theme: 'Fine Balance' and four subthemes: Complexity of selfneglect; Personal Health Care Professionals response to SN; Challenges in Managing the case; and, Recommendations for Practice. An effective approach to self-neglect was perceived by participants to be a fine balance from initial assessment to diagnosis, responses and management. Needs of the person were central to intervening effectively.

\section{PRACTICE WISDOM: PROFESSIONAL RESPONSES TO SELF-NEGLECT IN ISRAEL}

T. Band-Winterstein ${ }^{1}$, I. Doron ${ }^{1}$, S. Naim², 1. University of Haifa, Haifa, Israel, 2. Ben Gurion University of the Negev, Be'er Sheva, Israel

Self-neglect amongst older adults is a social and health phenomenon that is attracting increased research interest in recent years. Very little empirical attention has been devoted to evaluating intervention programmes in this field. The aim of this qualitative study was to explore the meaning attributed to elder self-neglect by social workers $(n=16)$ in their encounters with self-neglecting elders professionals $(\mathrm{n}=16)$ using a "practice wisdom" model. Data collected via in-depth semi-structured interviews, which were later transcribed and content was analyzed. Four key scenarios emerged: Immediate threat to life; potential future threat to life; avoiding deterioration in the absence of imminent risk and addressing environmental nuisance. In order to provide appropriate intervention, the social workers developed intervention strategies based on the tension and the need for balance between preserving autonomy, protecting human rights and respecting the older persons' wishes versus paternalism and client safety.

\section{PROMOTING ORGANIZATIONAL LEARNING IN SELF-NEGLECT: EVIDENCE FROM REVIEWS OF SERIOUS CASES}

S. Braye ${ }^{1}$, D. Orr ${ }^{1}$, M. Preston-Shoot ${ }^{2}$, 1. Social Work, University of Sussex, Haywards Heath, United Kingdom, 2. University of Bedfordshire, Luton, United Kingdom

When an adult experiencing abuse or neglect dies, English statute requires the Safeguarding Adults Board (the interagency adult protection committee) to conduct a case review establishing what went wrong, and to ensure that learning is applied to how organizations and professions work together. Over 60 review reports were analysed. Initial analysis of each case (characteristics, recommendations and themes) was followed by cross-case analysis to construct a systemic learning framework. Emergent learning themes related to four domains: the adult, the interprofessional team around the adult, the organisations surrounding the team, and the adult protection committee coordinating those organisations. Effective practice requires alignment between all domains, ensuring organizational support for trust-based relationships with individuals, strong legal literacy, detailed staff guidance and training, mechanisms for resolving differences, and arrangements for coordinating intervention. Reviews provide important learning for policy and practice development, adding to the evidence base on achieving positive outcomes in self-neglect work.

\section{SESSION 3125 (SYMPOSIUM)}

\section{INNOVATIVE USES OF TECHNOLOGY IN ASSESSING PHYSICAL PERFORMANCE OF OLDER PERSONS}

Chair: C.C. Quinn, University of Maryland School of

Medicine, Baltimore, Maryland

The purpose of this symposium is to present use of technology to translate the assessment of physical functioning in older adults to clinical practice. Previous studies confirm the relationship of physical performance measures to disability onset, hospitalization, nursing home admission, falls, mortality and other outcomes. While performance measures are being used more frequently in aging research, their uptake in clinical practice has been slow, in part because of the added burden in clinical care of geriatric patients. Using technology to improve the simplicity and efficiency of these measures could have a major impact on their use in clinical practice.

In this symposium Dr. Charlene Quinn, will provide an overview of the translation of technology to clinical care.

Dr. Jack Guralnik will describe the development of a smart phone app for the administration of the Short Physical Performance Battery, a widely used assessment of gait, strength and balance.

Dr. W. Jack Rejeski will present the mobility assessment tool-short form (MAT-sf) which uses video animations to improve accuracy and precision measurement of mobility.

Dr. Miriam Morey will discuss walking speed in the $6^{\text {th }}$ Vital Sign research project to establish community population walking speed norms and promote walking speed as a vital sign.

Dr. Lisa Barry will discuss use of an RFID (small electronic chip) device to measure gait speed in the clinic setting.

Dr. Antoine Piau will present the RESPECT study of a shoe insole to monitor frailty, assessing gait characteristics and transmit data for the use of patients and physicians.

\section{A SMARTPHONE APP FOR THE SHORT PHYSICAL PERFORMANCE BATTERY}

J.M. Guralnik ${ }^{1}$, D. Rooks ${ }^{2}$, T. Webb ${ }^{3}$, 1. University of Maryland School of Medicine, Chevy Chase, Maryland, 2. Novartis Institutes of Biomedical Research, Cambridge, Massachusetts, 3. GE Healthcare - Lunar, Madison, Wisconsin

The Short Physical Performance Battery (SPPB) is a standardized assessment of lower extremity physical performance. 